\title{
Influence of phenolics on epithelial barrier function and the expression of tight junction proteins in Caco-2 cells
}

\author{
P. Kullamethee, D. M. Commane and I. R. Rowland \\ Department of Food and Nutritional Sciences, University of Reading, P.O. Box 226, Reading RG6 6AP, UK
}

\begin{abstract}
There is ongoing interest in the potential biological activities of plant polyphenols in human health. Polyphenols and their metabolites may provide beneficial effects in the gastrointestinal tract, where they have been shown to protect against inflammatory disease. We present data from an in vitro model indicating that plant polyphenols may improve the integrity of the gut barrier. We studied the effects of phenolics and their metabolites on a Caco-2 model of gut barrier function measuring changes in transepithelial electrical resistance (TER) and changes in the expression of TJ gene mRNA and protein (Claudin-1 (CD-1), CD-4, Occludin (OC) and Zonula occludin (ZO)) via real time RT-PCR and western blots. We found that polyphenols generally improved barrier integrity. (Catechin $\gamma$ TER $+3.17 \%$, quercetin $\gamma$ TER $+3.35 \%$, kaempferol $\gamma$ TER $+5.19 \%$, and epicatechin $\gamma$ TER $+17.94 \%)$ as did the simple phenolic metabolites of these polyphenols (3-phenylpropionic acid $\gamma$ TER $+4.21 \%$, benzoic acid $\gamma$ TER $+4.57 \%$, phenylacetic acid $\gamma$ TER $+5.63 \%$ and Caffeic acid $\gamma$ TER $+14.91 \%$ ) (Results are reported for $10 \mu \mathrm{M}$ treatments after 24 hours). We also found that most of the tested compounds increased the transcriptional regulation of CD-1, CD-4, OC and ZO-1 relative to control. Phenylacetic acid, caffeic acid and quercetin induced approximate 0.3 -fold and 1-fold significant increase in CD-1 and CD-4 mRNA levels, respectively $(p<0.05)$. Epicatechin increased expression of OC mRNA, and the expression of ZO-1 was significantly increased by both phenylacetic acid and caffeic acid. In western blots the total protein expression of CD-1 was significantly higher than control after incubation with caffeic acid, quercetin and epicatechin around 3.7, 1.5 and 1.6 times, respectively $(p<0.05)$. Furthermore, the localisation of CD-1 to the cell membrane was studied, microscopic analysis of Caco-2 cell monolayers incubated with caffeic acid, demonstrated higher intensities of protein CD-1 around cell membranes which may explain the increased TJ function over untreated controls. These data suggest that polyphenols and their metabolites may enhance intestinal barrier function via increased transcriptional expression and localization of TJ related genes.
\end{abstract}

